\title{
Design, Simulation and Optimization of a Slotted Waveguide Array with Central Feed and Low Sidelobes
}

\author{
Lácides Ripoll-Solano
}

\author{
Luis Torres-Herrera
}

\author{
Manuel Sierra-Pérez
}

\begin{abstract}
Slotted-waveguide arrays (SWA) have been employed in radar, remote sensing, and communication applications where narrow-beam or shaped-beam patterns are required. One of the most common configurations for resonant SWA places longitudinal slots located in the broad face of a rectangular guide which is fed by one end and is shorted at the other. However, the relative bandwidth achieved in these arrays is about $1 \%$.

This paper present the design, optimization and simulation of a two-layer centrally-fed waveguide slot array antenna with ten slots $(2 \times 10)$ operating at $12 \mathrm{GHz}$, that achieves a bandwidth of $4 \%(440 \mathrm{MHz})$ at a return loss level of $-14 \mathrm{~dB}$. The design was a two-step process, first meeting the bandwidth requirement, and then a sidelobe level (SLL) of $20 \mathrm{~dB}$. To achieve the SLL, the excitation coefficients were improved with a least-square optimization approach. The resulting radiation diagram computed from the models run in Matlab were verified with CST Microwave Studio, which takes as input the physical dimensions of the array and the locations of the slots. The design requirements were satisfactorily met.
\end{abstract}

Keywords-antenna, slot, resonant, slotted-waveguide, bandwidth, optimization

\section{INTRODUCTION}

Slotted-waveguide arrays (SWA) have been employed in radar, remote sensing, and communication applications. Because of integrated low-loss feeds and radiating elements, these antennas exhibit high values of radiation efficiency. With the use of accurate design techniques and full-wave analysis software, it is possible to precisely control the aperture distribution of slot arrays while accomplishing a good input match [1].

One of the most common configurations for the radiant element places longitudinal slots located in the broad face of a rectangular guide that is shorted at one end and is fed through the other. The slots on the guide are arranged parallel to the axis of the guide, sequentially alternating on either side of the axis, and spaced by $\lambda_{g} / 2$, as shown in Fig. 1. This configuration is called resonant slotted waveguide antenna array, which are designed for broadside operation, albeit at the expense of a limited bandwidth of around $1 \%$ of the operating frequency [2].

For this reason, designers resort to several analysis in frequency domain in order to predict the bandwidth of the array and its trade-offs. Hamadallah [3] offers a method to analyze the frequency behavior of longitudinal slotted arrays in rectangular waveguides. Takeshima and Isogai [4] found a relation between the input stationary wave ratio (SWR), the number of slots $(\mathrm{N})$, and the bandwidth of a rectangular waveguide resonant array. Feeding from the center of the array instead of the more traditional approach that does it from one end was studied by Muller [5]. Results showed an improvement in bandwidth, but at the expense of a degradation in the radiation diagram together with a more complex design process.

In this paper, a two-layered centrally-fed waveguide slot array antenna with ten slots $(2 \times 10)$ is designed, optimized and simulated, in order to increase its reflection bandwidth. Because both ends of this waveguide antenna are shorted, the distance between the ends and the furthest elements are halved (see Fig.1.), leading to the expectation of better bandwidth. Results show that a bandwidth around $4 \%$ is achieved when the distance between the central radiating slots is greater than $\lambda_{g} / 2$. However, as this separation breaks the uniformity required to apply the basic excitation coefficients, new coefficients are obtained using an optimization that fits both the required bandwidth and sidelobe level (SLL). The tools used for the optimization and simulation to obtain the radiation diagrams were Matlab ${ }^{\mathrm{TM}}$ and CST Microwave Studio ${ }^{\mathrm{TM}}$, respectively. The rest of the paper is organized as follows. Sec. II explains the design methodology. Sec. III shows the final results, comparing the optimized radiation diagram with a simulation using CST Microwave Studio. Sec. IV concludes the paper.

\section{ANTENNA DESIGN}

The design specifications of the requested SWA are given in Table I. A linear array of radiating slots, centrally fed, with two layers of waveguides is selected. The slot spacing to produce a broadside beam is assumed equal to $\lambda_{g} / 2$. The array is made up of 1) the feeding waveguide, 2) the radiating waveguide, and 3 ) the radiating slots, as shown in Fig. 1. The first layer is the radiating waveguide. This is fed from the second layer (the feeding waveguide), through a coupling slot on the broad 


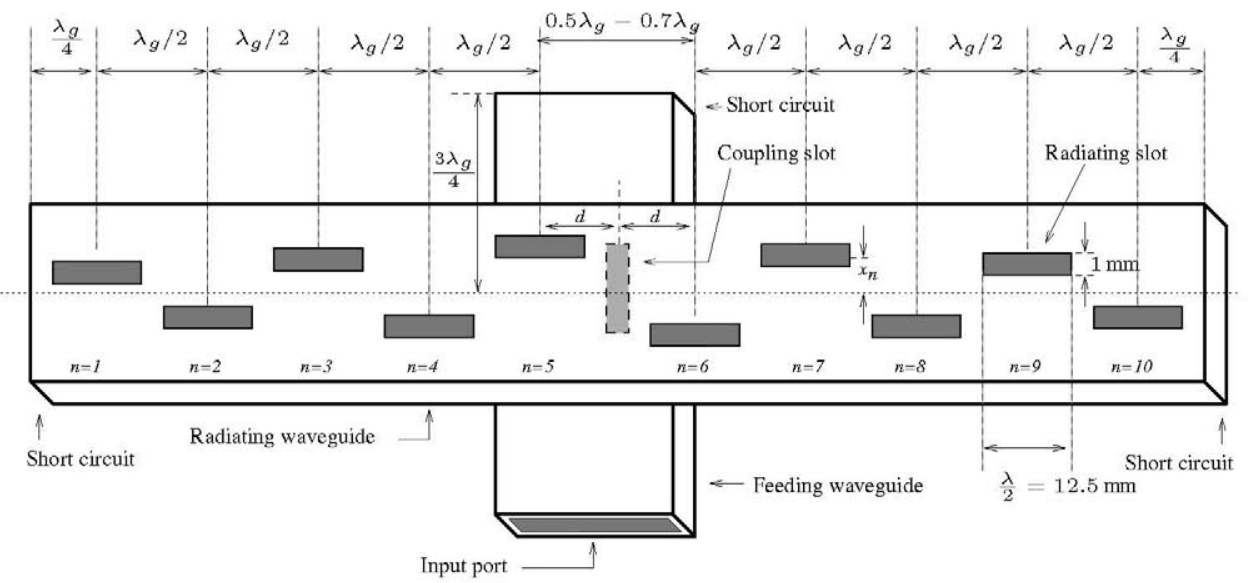

Fig. 1: Selected design for the slotted waveguide array.

TABLE I: ANTENNA DESIGN SPECIFICATIONS

\begin{tabular}{|c|c|}
\hline Parameter & Value \\
\hline \hline Frequency & $12 \mathrm{GHz}$ \\
\hline Minimum gain & $16 \mathrm{dBi}$ \\
\hline Beamwidth & $8^{\circ}$ (horizontal plane) \\
\hline Sidelobe level (SLL) & $20 \mathrm{~dB}$ \\
\hline Type of radiation & Broadside \\
\hline Minimum efficiency & $55 \%$ \\
\hline Polarization & Horizontal \\
\hline Minimum relative bandwidth & $\approx 4 \%$ \\
\hline Return loss level for bandwidth & $14 \mathrm{~dB}$ \\
\hline
\end{tabular}

wall of the waveguides connecting both layers. The coupling slot has an offset from the center of the feeding waveguide, thus acting as an impedance transformer between the two layers. The symmetrical placement of the radiating slots at both sides of the coupling slot, and the constant separation of $\lambda_{g} / 2$ between them, produces a $180^{\circ}$ phase shift in each slot, which together with the resonant wave inside the guide, causes that all slots radiate in-phase. Finally, the feeding waveguide is $3 \lambda_{g} / 4$ long measured from the longitudinal axis of the radiating waveguide to the short-circuited end of the feeding waveguide. Also, short-circuiting both ends of the radiating waveguide and placing the last radiating slots to a distance of $\lambda_{g} / 4$ from each end contributes to a better resonance.

\section{A. Waveguide selection and number of array elements}

Based on the requirements such as operation frequency, beamwidth, SLL and bandwidth, the type of waveguide is selected: WR-75. This waveguide has an inner width of $19.05 \mathrm{~mm}$, and inner height of $9.5 \mathrm{~mm}$, which at $12 \mathrm{GHz}$ results in a in-guide wavelength $\lambda_{g}=3.313 \mathrm{~cm}$.

The number of radiating elements (slots) in the array is computed based on Mehmood and Yu [6] as follows:

$$
N=\left\lceil 50.77 \frac{\lambda}{B W \cdot s}\right\rceil
$$

where $[\cdot]$ is the integer greater than and closest to the argument, $\mathrm{N}$ is the number of slots, $\lambda$ is the free space wavelength at $12 \mathrm{GHz}$ in $\mathrm{cm}(\lambda=2.5), B W$ is the beamwidth $\left(B W=8^{\circ}\right)$, and $s$ is the inter-slot separation along the longitudinal axis $\left(s=\lambda_{g} / 2\right)$. The computation in $N=\lceil 9.57\rceil=10$ slots.

\section{B. Theoretical radiation diagram}

The next step is the computation of the theoretical radiation diagram based on the traditional (uniformly spaced) slots. For this, excitation coefficients are obtained with the DolphChebyshev method for $N=10$ and $S L L=20 \mathrm{~dB}$. These are shown as $a_{n}$ in Table II. Finally, the theoretical diagram, shown in Fig. 2, corresponds to the normalized magnitude of the complex radiated electrical field, given by:

$$
E(\theta)=f(\theta) \sum_{n=1}^{N} a_{n} e^{j n k \frac{\lambda_{g}}{2} \cos \theta}
$$

where $k=\frac{2 \pi}{\lambda}$ is the wave number of free space, $\theta$ is the angle with respect to the array axis, and $f(\theta)$ es the electrical field radiated by a single array element, that is, one slot of length $\lambda / 2=12.5 \mathrm{~mm}$ and width $1 \mathrm{~mm}$, which is equivalent to a dipole of similar length, and computed as:

$$
f(\theta)=\frac{\cos \left(\frac{\pi}{2} \cos \theta\right)}{\sin \theta}
$$

\section{Slot locations on the waveguide}

Subsequently, with the theoretical excitation coefficients, the separation $x_{n}$ (see Fig. 1) of the slots with respect to the longitudinal axis of the waveguide are obtained. To this end, an intermediate step is carried out, where the conductances $g_{n}$ of an electrical equivalent of the antenna (based on transmission line theory) are given by:

$$
g_{n}=\frac{a_{n}^{2}}{\sum_{n=1}^{N / 2} a_{n}^{2}}
$$




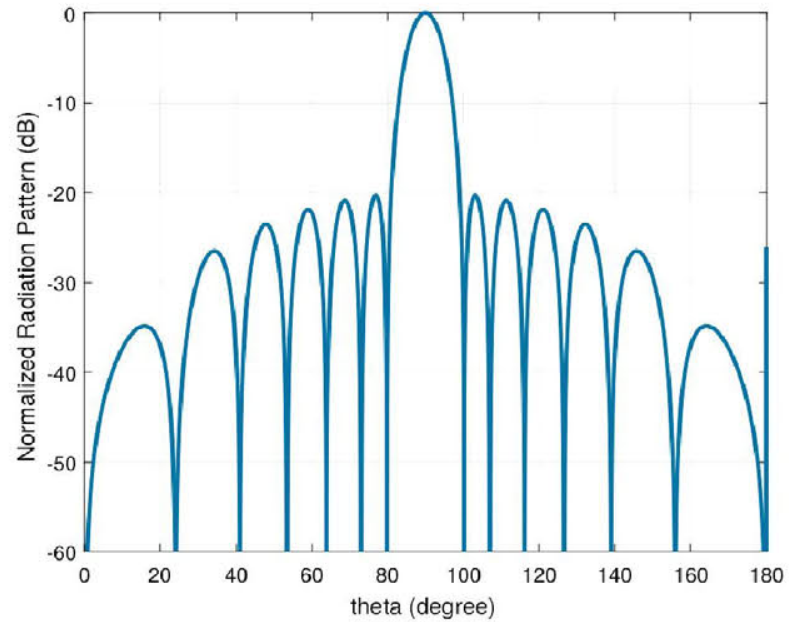

Fig. 2: Theoretical radiation diagram for uniformly spaced slots by $\lambda_{g} / 2$ (Plane $\phi=90^{\circ}$ ).

TABLE II: NORMALIZED COEFFICIENTS, CONDUCTANCES AND SEPARATIONS SLOT-AXIS

\begin{tabular}{|c|c|c|c|}
\hline Slot & $a_{n}$ & $g_{n}$ & $x_{n}(\mathrm{~mm})$ \\
\hline \hline 1 and 10 & 0.6416 & 0.1279 & 2.5192 \\
\hline 2 and 9 & 0.5944 & 0.1098 & 2.3238 \\
\hline 3 and 8 & 0.7780 & 0.1880 & 3.1009 \\
\hline 4 and 7 & 0.9214 & 0.2658 & 3.7486 \\
\hline 5 and 6 & 1.0000 & 0.3106 & 4.1252 \\
\hline
\end{tabular}

The conductances $g_{n}$ and the slot location offsets $x_{n}$ are listed in Table II. The offsets $x_{n}$ are related to $g_{n}$ by:

$$
g_{n}=2.09 \frac{\lambda_{g}}{\lambda} \frac{a}{b} \cos ^{2}\left(\frac{\pi \lambda}{2 \lambda_{g}}\right) \sin ^{2}\left(\frac{\pi x_{n}}{a}\right)
$$

where $a$ and $b$ are the inner width and height of the rectangular waveguide, respectively.

As the next step in the design, the location of coupling slot will be optimized. The separation between the two central radiating slots was hold $\lambda_{g} / 2$, and the location of the coupling slot was varied. Using the CST Microwave Studio ${ }^{\mathrm{TM}}$, the best location of the coupling slot was found at $7 \mathrm{~mm}$ from the center of the feeding waveguide, resulting in $14 \mathrm{~dB}$ return losses, in the widest found range from $11.9 \mathrm{GHz}$ to $12.04 \mathrm{GHz}$ $(\approx 1.2 \%)$, as shown in Fig. 3. The design committed to the location found for the coupling slot, although the bandwidth requirement of $\approx 4 \%$ was not yet met.

It was found that the distance between the two central radiating slots, which is related to the mutual coupling degree and to the coupling slot, has the most influence on the bandwidth. Weakening this coupling, revealed an increase in bandwidth. Therefore, the two sets of radiating slots at both sides of the coupling slot, were moved off the center, but keeping the other inter-slot distance fixed at $\lambda_{g} / 2$. Thus, when the separation between the two central radiating slots reached $0.7 \lambda_{g}$, the best bandwidth was attained for a $14 \mathrm{~dB}$ return loss,

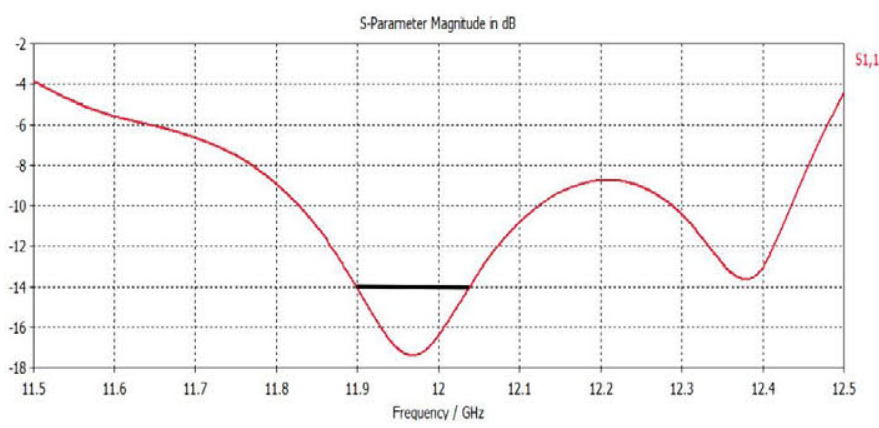

Fig. 3: Return loss in $\mathrm{dB}\left(S_{11}\right)$ for a separation between the two central radiating slots $d=0.5 \lambda_{g}$

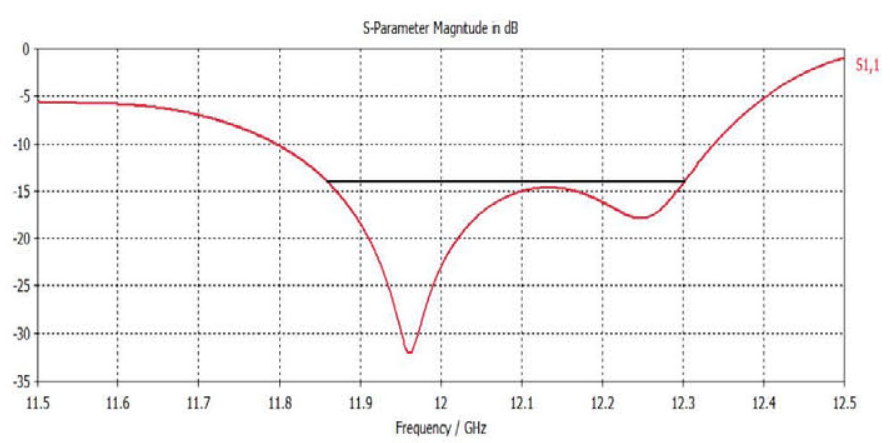

Fig. 4: Return loss in $\mathrm{dB}\left(S_{11}\right)$ for a separation between the two central radiating slots $d=0.7 \lambda_{g}$

as shown in Fig. 4. The bandwidth is approximately $440 \mathrm{MHz}$, meeting the requirement of being close to $4 \%$ given in Table I.

\section{Coefficient optimization to attain SLL}

At this point of the design, the geometrical construction of the antenna is completely defined. Also, the requirements of beamwidth, bandwidth, return loss, broadside radiation are all met. On the other hand, the non uniform spacing of the radiating slots in the array deteriorates the sidelobe level, which is not reaching the required $20 \mathrm{~dB}$. The next step is to recover the desired SLL. The way to tackle this problem is to recall that the radiation diagram comes from the excitation coefficients. Unfortunately, the coefficients are obtained under the assumption of the Dolph-Chebyshev method, where the inter-slot distances are uniform. This no longer holds in the current design, where the two central slots are separated by $0.7 \lambda_{g}$, instead of $0.5 \lambda_{g}$ used by the rest.

For this reason, an optimization over the excitation coefficient is carried out, taking as starting point the original coefficients $a_{n}$ and obtaining a new optimized set called $a_{n}^{o p t}$. The optimization uses Least Squares method to place the normalized secondary-lobe maximums of the radiation diagram $20 \mathrm{~dB}$ lower than the main lobe maximum, set at $0 \mathrm{~dB}$.

The objective function is the error between the $-20 \mathrm{~dB}$ target value and the actual value attained at each maximum at each secondary lobe. The problem is formulated as follows: 


$$
\begin{gathered}
\underset{\left(a_{1}, a_{2}, \ldots, a_{N}\right)}{\operatorname{minimize}} \sum_{i=1}^{M_{S L}} \epsilon_{i}^{2} \\
\forall i: \epsilon_{i}=20 \log _{10}\left|\hat{F}\left(\theta_{i}\right)\right|-S L L_{i} \\
\forall i: \hat{F}\left(\theta_{i}\right)=F\left(\theta_{i}\right) / F(0) \\
F(\theta)=f(\theta) \sum_{n=\frac{N}{2}+1}^{N} a_{n} \operatorname{Re}\left\{e^{j k\left(d+(n-1) \frac{\lambda_{g}}{2}\right) \cos \theta}\right\} \\
\forall i, \theta>90^{\circ}:\left\{\theta_{i}\right\}=\left\{\theta \mid \operatorname{roots} \text { of } \frac{\delta F}{\delta \theta}=0\right\}
\end{gathered}
$$

where $i$ is the index for most relevant sidelobes, which is limited to the first five, i.e., $M_{S L}=5$. Moreover, $S L L_{i}$ is the target value at sidelobe $i$, being typically $-20 \mathrm{~dB} . f(\theta)$ as defined in Eq. (3), $\theta_{i}$ are the angles at which the sidelobes attain a maximum, and $d$ is half the distance between the central radiating slots, i.e. $d=\frac{0.7 \lambda_{g}}{2}$.

This non convex and non analytic optimization was done in Matlab ${ }^{\mathrm{TM}}$, starting at the original value of the excitation coefficients $a_{n}$ from Table II, and then producing the new set of optimized coefficients $a_{n}^{o p t}$, shown in Table III. The table also shows the optimized conductances $g_{n}^{o p t}$ and offsets $x_{n}^{o p t}$.

TABLE III: OPTIMIZED COEFFICIENTS, CONDUCTANCES AND SEPARATIONS SLOT-AXIS

\begin{tabular}{|c|c|c|c|}
\hline Slot & $a_{n}^{\text {opt }}$ & $g_{n}^{\text {opt }}$ & $x_{n}^{\text {opt }}(\mathrm{mm})$ \\
\hline \hline 1 and 10 & 0.6725 & 0.1713720 & 2.9474 \\
\hline 2 and 9 & 0.4199 & 0.0667910 & 1.7948 \\
\hline 3 and 8 & 0.7305 & 0.2021466 & 3.2272 \\
\hline 4 and 7 & 0.6910 & 0.1808765 & 3.0359 \\
\hline 5 and 6 & 1.0000 & 0.3788140 & 4.6566 \\
\hline
\end{tabular}

\section{RESULTS}

After the design process, its quality is quantified verifying the fulfillment of all requirements. In the first part, the main goals were to ensure 1) the minimum antenna gain, which is related to the beamwidth, and 2 ) the $\approx 4 \%$ bandwidth measured at the $14 \mathrm{~dB}$ return loss level. Both goals were attained with $N=10$ slots and an non uniform element placement in the array. As a consequence of this, the DolphChebyshev method could not be applied to get the excitation coefficients. Therefore, a new set was obtained optimizing the SLL as a function of such coefficients. With the new values, the new slot offsets in the waveguide were computed.

Finally, the radiation diagram can be verified by contrasting the behavior of the optimized field intensity of Eq.(6d) with that obtained by simulation. The simulation is done in CST Microwave Studio, which takes as input a physical model of the antenna, according to the computed slot locations, dimensions and coupling between the two layers and produces its corresponding radiation diagram. The result is shown in Fig. 5, where the optimized diagram mostly meets the requirements, improving over the original diagram specially

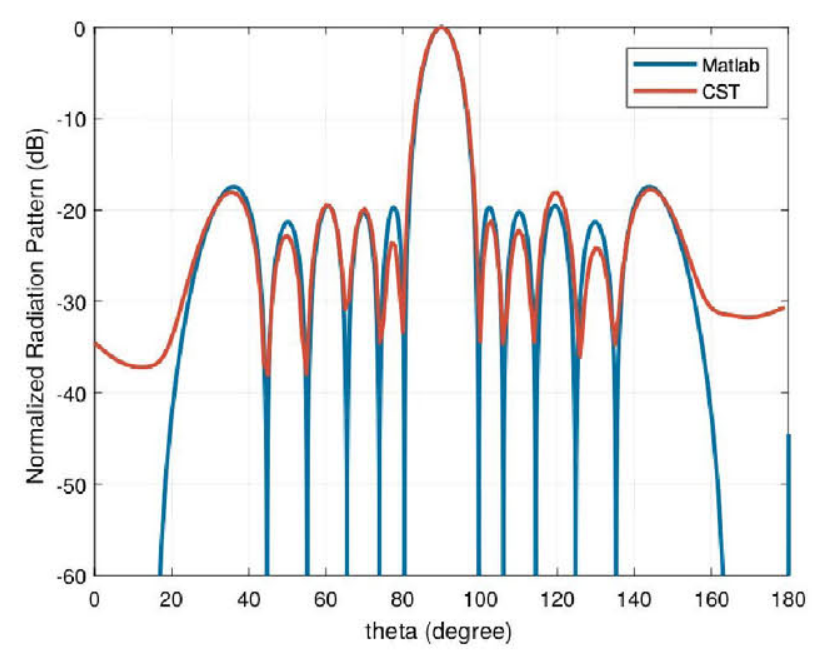

Fig. 5: Radiation diagram obtained with the model run in Matlab and the CST Microwave Studio

at the first and third sidelobes. On the other hand, at the fifth sidelobe $\left(\approx 145^{\circ}\right)$ of the optimized diagram there is a small violation of the $S L L$ requirement by around $2 \mathrm{~dB}$. The influence of this sidelobe at that direction is not critical.

\section{CONCLUSIONS}

The design methodology of a linear slotted waveguide array with central feed and low sidelobes at $12 \mathrm{GHz}$ was presented. The process targets two main requirements: the bandwidth and the sidelobe level. Starting with the basic configuration of uniformly spaced slots, the goal of increasing the bandwidth to $\approx 4 \%$, i.e. $440 \mathrm{MHz}$, at a return loss level of $-14 \mathrm{~dB}$ with respect to the most common $1 \%$ in lateral-fed guides, was achieved. The non uniformity in the slot placement called for an optimization of the excitation coefficients, which determined the slot offsets with respect to the waveguide longitudinal axis. Once this was tuned, the SLL of $20 \mathrm{~dB}$ requirement was finally met.

\section{REFERENCES}

[1] L. Ripoll, D. Pardo, and M. Sierra, "Diseño, simulación y construcción de un subarray para una antena de ranuras de comunicaciones por satélite," Interciencia, vol. 40, no. 7, pp. 487-491, Jul. 2015.

[2] L. Ripoll, M. Sierra, and D. Pardo, "Design, simulation and test of a slot antenna array using one parameter Taylor synthesis in the $\mathrm{GHz}$ range," IEEE Latin America Trans., vol. 13, no. 10, pp. 3210-3215, Oct. 2015.

[3] M. Hamadallah, "Analysis of frequency behavior of slot arrays," Proc. 1987 IEEE Int. Symp. Antennas Propagat., pp. 306-309, 1987.

[4] T. Takeshima and Y. Isogai, "Frequency bandwidth of slotted array aeria system," Electron. Eng., pp. 201-204, Feb. 1969.

[5] M. Muller, I. Theron, and D. Davidson, "Improving the bandwidth of a slotted waveguide array by using a center-feed configuration," Proc. 1999 AFRICON, pp. 1075-1080, 1999.

[6] T. Mehmood and Y. Yu, "Design of a novel flat array antenna for radio communications," Master of Science in Electrical Engineering, Blekinge Institute of Technology, Sweden, 2011. 